\title{
Extensions and Index of Hermitian Representations
}

\author{
By
}

\author{
Palle E.T. JORGENSEN*
}

Table of Contents
$\S 1$. Introduction
\$2. Definitions
§3. Preliminary Results
$\S 4$. Irreducible Modules
§5. Selfadjoint Representations
$\S 6$. Relatively Bounded Representations
§7. References

\begin{abstract}
We study Hermitian representations of algebras $\boldsymbol{A}$ with involution, $a \rightarrow a^{*}$. A representation $\pi$ of $\boldsymbol{A}$ on a vector space $D$ is said to be Hermitian (or to be a positive-energyrepresentation) if $D$ carries a positive definite inner product $(\cdot, \cdot)$ such that

$$
(\pi(a) u, v)=\left(u, \pi\left(a^{*}\right) v\right), \quad a \in \boldsymbol{A}, u, v \in D .
$$

Such representations arise in quantum field theory and in the study of unitary representations of Lie groups. They were introduced (in this general context) by Powers. We show that main features of von Neumann's index theory (for single Hermitian operators) carry over to representations. Moreover, we get explicit index-information directly from the representation theory, and this is applied to the study of representations of semisimple Lie algebras $g$ (with Cartan decomposition $g=\mathfrak{f}+\mathfrak{p}$ ).

For a certain class (to be specified below) of positive energy representations $\pi$, we show that the index may be computed from the restriction of $\pi$ to the compact subalgebra 1 . Our results are then applied to the integrability problem for representations of semi-simple Lie algebras.

Several classes of examples are included.
\end{abstract}

\section{§1. Introduction}

In this paper, we study Hermitian representations of algebras with involu-

Communicated by H. Araki, December 26, 1988. Revised April 25, 1989.

* Department of Mathematics, The University of Iowa, Iowa City, IA 52242 U.S.A.

** Research supported in part by NSF. 
tion and give applications to representations of Lie algebras. We are motivated by von Neumann's classification of extensions of single Hermitian operators with dense domain in a given Hilbert space. We show that the extension theory for Hermitain representations is somewhat analogous to the case of a single Hermitain operator. Using Powers' definition [Po] of the adjoint representation we identify a generic quadratic form on the domain of the adjoint representation. If $\pi$ is the given Hermitian representation with adjoint $\pi^{*}$, we consider the quotient $D\left(\pi^{*}\right) / D(\pi)$ where $D\left(\pi^{*}\right)$, resp. $D(\pi)$, denote the corresponding domains. We characterize the Hermitian extensions, and the selfadjoint representations in terms of the quadratic form (the boundary form), and consider the decomposition theory for the induced module $D\left(\pi^{*}\right) / D(\pi)$.

We apply the results to the special case where the algebra is the universal enveloping algebra of a given semisimple Lie algebra g. For a given Cartan decomposition $g=\mathfrak{f}$ and a quasi-simple Hermitian representation $\pi$, we show that the boundary module is given by the restriction to the representation. We finally apply this result to the integrability question (see [JMo]) for representations of $\mathbb{L i e}$ algebras.

We study the canonical quadratic form on a certain induced module associated with a given Hermitian representation.

Since the quadratic form is not positive definite, standard results from Hilbert space theory on decomposition of modules do not apply in the present setting. Instead we are motivated by the Gupta-Bleuler theory of module triples associated with invariant (nonpositive definite) inner products.

The reader is referred to [Ar], [Helt], [In], [ $\mathrm{Kr}],[\mathrm{Na}],[\mathrm{Ot}]$, and [Ta] for further details on this last point. The recent paper [Ha] has further results in a restricted context.

Our motivation comes primarily from the idea of Powers to adapt von Neumann's index for Hermitian unbounded operators to representation theory $[\mathbb{P o}]$. We note that this leads to an induced module, associated with a given Hermitian representation of the given algebra with involution. This module carries a family of quadratic forms indexed by the algebra, and the GuptaBleuler approach may be adapted to this family of quadratic forms.

Hermitian representations have also been considered earlier in a general context, see e.g. [In], [Jo (a)-(c)], [Ne], [Ph], and [Ra]. We further mention the recent idea of Powers-Price [PP] to consider induced modules (analogous to our modules $D\left(\pi^{*}\right) /(D(\pi))$ in connection with an index theory for semigroups of endomorphisms of $B(H)$, see also [Arv]. 
It is our hope that the ideas on index may be adapted to the general study of derivations in $C^{*}$-algebras [GJ], and representations by Lie algebras as derivations $[\mathrm{Ot}]$ as they arise in noncommutative differential geometry.

Finally we point out in [Jo (c)] that the question of finding the unitarizable representations of infinite dimensional Lie algebras (such as the Virasoro algebra [GW], [CP]) may be phrased in terms of Hermitian representations of associative algebras with involution. In this case, the inner product is not given $a$ priori which adds one difficult element to the problem. For a given algebra $\boldsymbol{A}$, and a representation $\pi$ of $\boldsymbol{A}$ with domain $D$, the object is to find a positive definite inner product $(\cdot, \cdot)$ on $D$ such that

$$
(\pi(a) u, v)=\left(u, \pi\left(a^{*}\right) v\right), \quad a \in \boldsymbol{A}, u, v \in D .
$$

\section{$\S 2$. Definitions}

Consider a given Hilbert space $H$ with inner product written $(\cdot, \cdot)$, and let $D$ be a dense linear subspace of $H$. Let $\boldsymbol{A}$ be a given (associative) algebra with involution, written $a \rightarrow a^{*}$. We say that $\pi$ is a representation of $\boldsymbol{A}$ with $D$ as domain if $\pi$ is a representation of $\boldsymbol{A}$ by linear operators $\pi(a)$ such that, for all $a \in \boldsymbol{A}$, the operator $\pi(a)$ is defined on $D$ and maps $D$ into itself.

We say that $\pi$ is Hermitian if

$$
(\pi(a) u, v)=\left(u, \pi\left(a^{*}\right) v\right), \quad a \in \boldsymbol{A}, u, v \in D .
$$

2.1. Let $\pi$ be a given Hermitian representation on an algebra $\boldsymbol{A}$ with *-involution. Assume that the domain $D$ of $\pi$ is dense in the representation space, a given Hilbert space $H$, and let $\pi^{*}$ denote the dual representation with domain $D^{*}$, defined as follows:

$$
D^{*}=\cap\left\{\operatorname{dom}\left(\pi(a)^{*}\right): a \in \boldsymbol{A}\right\}
$$

with

$$
\pi^{*}(a):=\left.\pi\left(a^{*}\right)^{*}\right|_{D^{*}}, \quad a \in \boldsymbol{A} .
$$

Since $\pi$ is Hermitian, for each $a \in \boldsymbol{A}, \pi(a)$ is contained in the operator $\pi\left(a^{*}\right)^{*}$, i.e.,

$$
\pi(a) \subset \pi\left(a^{*}\right)^{*},
$$

and it follows that each $\pi(a)$ is closable. Let $\overline{\pi(a)}$ denote the closure, i.e, the smallest closed operator containing $\pi(a)$. Then

$$
\overline{\pi(a)} \subset \pi\left(a^{*}\right)^{*} \text {. }
$$


We define the closure of $\pi$ as $\bar{\pi}$ by the following: The domain of $\bar{\pi}$ is defined by

$$
\bar{D}:=\cap\{\operatorname{dom}(\overline{\pi(a)}): a \in A\}
$$

and

$$
\bar{\pi}(a)=\left.\pi^{*}(a)\right|_{\bar{D}}=\left.\overline{\pi(a)}\right|_{\bar{D}} .
$$

Powers [ $[\mathrm{Po} \mathbb{I}]$ showed that $\bar{\pi}$ is a Hermitian representation. Moreover, if $\rho$ is an arbitrary Hermitian representation which extends $\pi$, then

$$
\rho \subset \pi^{*} \text {. }
$$

If $\rho$ is an extension of $\pi$ which is both Hermitian and closed (i.e., $\bar{\rho}=\rho$ ), then

$$
\bar{\pi} \subset \rho \subset \pi^{*} \text {. }
$$

2.2. The boundary conditions of a given Hermitian representation $\pi$ are defined in terms of the following trilinear form (called the deficiency form) on $A \times D^{*} \times D^{*}, B(a, u, v)$ given for $a \in A$ and $u, v \in D^{*}$ by:

$$
B(a, u, v)=\left(\pi^{*}(a) u, v\right)-\left(u, \pi(a)^{*} v\right)
$$

where $(\circ, \circ)$ denotes the given positive definite inner product on $H$. The inner product is assumed to be sesquilinear in the two variables with linearity in the second variable. It follows that $B$ is linear in the first and the last variable, and conjugate linear in the middle variable.

A linear subspace $E$ is said to be intermediate if

$$
D \subset E \subset D^{*} \text {. }
$$

An intermediate subspace $E$ is said to be symmetric if

$$
B(a, u, v) \equiv 0, \quad \forall a \in A, \quad u, v \in E .
$$

We say that $E$ is a $\pi^{*}$-module, or jusí an $\mathbb{A}$-module if

$$
\pi^{*}(a) E \subset \mathbb{E}, \quad a \in A,
$$

Note that the domain $D$ of $\pi$ is intermediate, symmetric and a $\pi^{*}$-module.

2.3. We say that a given Hermitian representation $\pi$ is closed if $\pi=\bar{\pi}$ when $\bar{\pi}$ is defined as in 2.1 above. We say that $\pi$ is selfadjoint if $\pi=\pi^{*}$ where the dual representation $\pi^{*}$ is also defined in 2.1 above. Finally, $\pi$ is said to be essentially selfadjoint if

$$
\bar{\pi}=\pi^{*} \text {. }
$$


2.4. A given Hermitian representation $\pi$ with dense domain $D$ is said to be nondegenerate if the family $\{B(a, \cdot, \cdot): a \in \boldsymbol{A}\}$ of sesquilinear forms separates points on the quotient space $D^{*} / D$. Specifically, if $u \in D^{*}$ satisfies $B(a, u, v)=0$ for all $a \in A$ and $v \in D^{*}$, then it follows that $u \in D$.

Note that, since $\pi$ is Hermitian, the deficiency forms $B(a, \cdot, \bullet)$ pass to the quotient to define sesquilinear forms on $\left(D^{*} / D\right) \times\left(D^{*} / D\right)$ where $D^{*}$ denotes the domain of the dual representation $\pi^{*}$. Also note that $B(a, \cdot, \cdot)$ is generally not positive definite. When $\boldsymbol{a} \in \boldsymbol{A}$ is given, $B(a, \cdot, \cdot)$ may be viewed as a sesquilinear form on $D^{*} / D$ but it need not be nondegenerate even if $\pi$ is assumed nondegenerate. But if $\pi$ is nondegenerate, and if $\xi \in D^{*} / D$ satisfies $B(a, \xi, \eta)=0$ for all $a \in \mathbb{A}$ and $\eta \in D^{*} / D$, then it follows that $\xi$ is the zero-vector in $D^{*} / D$.

2.5. The domain $D^{*}$ of the dual representation $\pi^{*}$ acquires a topology from the family of seminorms $\left\{p_{a}: a \in \mathbb{A}\right\}$ defined as follows:

$$
p_{a}(u):=\left\|\pi^{*}(a) u\right\|, \quad u \in D^{*} .
$$

An intermediate subspace $E$ is said to be closed if it is closed relative to this locally convex topology given by the seminorms $\left\{p_{a}: a \in \mathbb{A}\right\}$ on $D^{*}$.

\section{§3. Preliminary Results}

In this section, we show that extensions of a given Hermitian representation may be classified in a manner which is quite analogous to the classification, due to von Neumann ([vN], [DS]), of extensions of a single Hermitian symmetric operator with dense domain in a given Hilbert space.

Given, at the outset, will be a Hermitian representation $\pi$ with dense domain, and the corresponding (dual) adjoint representation (introduced first by Powers [Poll]) will be denoted $\pi^{*}$.

Lemma 3.1. Let $\pi$ be a Hermitian representation of a given algebra $\mathbb{A}$ with $*$-involution. Let $D$ be the domain of $\pi$, and let $\pi *$ be the dual representation with domain $D^{*}$

Then there is a 1-1 correspondence between Hermitian extension representations $\rho$ and intermediate subspaces $E$ such that

(i) $E$ is symmetric and

(ii) $E$ is a $\pi^{*}$-module.

Moreover, the intermediate subspace $E$ is closed $f$ and only if the corresponding representation $\rho$ is closed. 
If $\rho$ is a given Hermitian extension representation, then we define the corresponding intermediate subspace $E$ by

$$
E:=\operatorname{dom}(\rho),
$$

If, conversely, an intermediate subspace $E$ is given, satisfying conditions (i) and (ii), then define, for $a \in \mathbb{A}$,

$$
\rho(a):=\left.\pi^{*}(a)\right|_{E} .
$$

Proof. Immediate!

Lemma 3.2. Let $\pi$ be a Hermitian representation, and let $E$ be an intermediate subspace which is a $\pi^{*}$-module.

Then the dual subspace $E^{\perp}$ defined by

$$
E^{\perp}=\left\{u \in D^{*}: B(a, u, v)=0 \forall a \in \mathbb{A}, v \in E\right\}
$$

is also a $\pi^{*}$-module, i.e., $\pi^{*}(a)\left(E^{\perp}\right) \subset E^{\perp}$ for all $a \in \mathbb{A}$.

Proof. We shall need the following formula for the deficiency form $B$. Recall $B(a, u, v)$ is defined for $a \in \mathbb{A}$ and $u, v \in D^{*}$ by (2.9). Let $a, b \in \mathbb{A}$. We claim that

$$
B(a b, u, v)=B\left(a, \pi^{*}(b) u, v\right)+B\left(b, u, \pi(a)^{*} v\right) .
$$

We check this by computing the right hand side, substituting (2.9);

$$
\begin{aligned}
B(a, & \left.\pi^{*}(b) u, v\right)+B\left(b, u, \pi(a)^{*} v\right) \\
& =\left(\pi^{*}(a) \pi^{*}(b) u, v\right)-\left(\pi^{*}(b) u, \pi(a)^{*} v\right)+\left(\pi^{*}(b) u, \pi(a)^{*} v\right)-\left(u, \pi(b)^{*} \pi(a)^{*} v\right) \\
& =\left(\pi^{*}(a b) u, v\right)-\left(u, \pi(a b)^{*} v\right) \\
& =B(a b, u, v)
\end{aligned}
$$

which concludes the proof of formula (3.4).

We now utilize (3.4) as follows: Let $u \in E^{\perp}$ and $b \in A$ be given. It is assumed that $E$ is a $\pi^{*}$-module, and we claim that $\pi^{*}(b) u \in E^{\perp}$. We note that the two terms $B(a b, u, v)$ and $B\left(b, u, \pi(a)^{*} v\right)$ from (3.4) both vanish whenever $a \in \mathbb{A}$ and $v \in E$. For $B(a b, u, v)$ this follows since $u \in E^{\perp}$, and, for the second term $B\left(b, u, \pi(a)^{*} v\right)$, we note that $\pi(a)^{*} v=\pi^{*}\left(a^{*}\right) v \in E$ since $v \in E$ and $E$ is a $\pi^{*}$-module. Hence the third term $B\left(a, \pi^{*}(b) u, v\right)$ must vanish. Since $a \in \mathbb{A}$ and $v \in E$ are arbitrary, we finally conclude that $\pi^{*}(b) u \in E^{\perp}$, which is the desired conclusion.

Let $\pi$ be a given closed Hermitian representation. 
We now proceed to prove two results which allow us to check when a given intermediate subspace $E$ is symmetric. We shall assume that $E$ is a $\pi^{*}$-module. The condition for $E$ to be symmetric amounts to a complicated system of linear equations. In the results, we show how to get symmetric subspaces from algebraic conditions on $E$. We shall be concerned with irreducibility conditions.

In the first lemma, we shall assume that $\pi^{*}(\mathbb{A})$ acts irreducibly on $E$, and in the second lemma we shall pass to the quotient $D^{*} / D$ (i.e., the induced module) and consider an irreducibility condition for the quotient-representation.

We have seen that both the representation $\pi^{*}$, and the deficiency form $B$, pass to $D^{*} / D$. For $\pi^{*}$, this follows from the two properties:

$$
\pi(a) D \subset D \text { and } \pi=\left.\pi^{*}\right|_{D}
$$

For $B$, it follows from the fact that $B(a, \cdot, \cdot)$ vanishes identically on $D \times D$ for all $a \in \mathbb{A}$.

We shall consider $D^{*}$ as a topological linear space with the topology determined by the seminorms from (2.14). If $D \subset E \subset D^{*}$, we say that $E$ is irreducible if $E$ is an irreducible $\pi^{*}$-module, i.e., it has no closed invariant subspaces other than $D$ and $E$ itself.

Let $Q: D^{*} \rightarrow D^{*} / D=M$ denote the quotient mapping, and let $D^{*} / D$ acquire the corresponding projective topology. We say that a subspace $F \subset M:=D^{*} / D$ is irreducible if $F$ is an irreducible $\pi^{*}$-module. We assume that the induced action of $\boldsymbol{A}$ on the quotient does not have any nontrivial invariant subspaces of $F$, i.e., no invariant subspaces of $F$ other than 0 and $F$ itself.

With the definition of the quotient space $M=D^{*} / D$, and the action of $\mathbb{A}$ on $M$ induced from the action of $\pi^{*}$ on $D^{*}$, we get a one-to-one correspondence between irreducible closed intermediate modules $E$ and irreducible modules $F$ in the quotient $M$ : If $E$ is a given irreducible intermediate module, then $F=Q E$ is an irreducible module in the quotient, and every irreducible module in the quotient is of this form. If, conversely, $F$ is given, we may take $E=Q^{-1}(F)$.

\section{$\S 4$. Trreducible Modules}

We now show that representation theory applies directly to the problem of finding Hermitian extensions of a given Hermitian representation.

Proposition 4. 1 . Let $\pi$ be a closed Hermitian representation, and let $E$ be an intermediate subspace which is also an irreducible $\pi^{*}$-module. 
Then it follows that $D \subset E^{\perp}$, and $E$ is symmetric if and only if $E \cap E^{\perp} \neq D$ where $D$ is the domain of $\pi$ and

$$
E^{\perp}=\left\{u \in D^{*}: B(a, u, v)=0 \forall a \in A, v \in E\right\} .
$$

Proof. We first prove the inclusion $D \subset E^{\perp}$. Let $a \in A, u \in D$ and $v \in E$. We must show that $B(a, u, v)=0$. But $B(a, u, v)=\left(\pi^{*}(a) u, v\right)-\left(u, \pi(a)^{*} v\right)$, and $\pi(a) \subset \pi^{*}(a)=\pi\left(a^{*}\right)^{*}$. Since $u \in D$, we get

$$
\begin{aligned}
B(a, u, v) & =(\pi(a) u, v)-\left(u, \pi(a)^{*} v\right) \\
& =\left(u, \pi(a)^{*} v\right)-\left(u, \pi(a)^{*} v\right) \\
& =0,
\end{aligned}
$$

proving the assertion.

Since $E$ is a module, it follows that $E^{\perp}$ is also a module. Consider $K:=E \cap E^{\perp}$. We have $D \subset E \subset D^{*}$, and $D \subset K \subset E$, and moreover $K$ is a closed module. To get $K=E$, we need only rule out the possibility $K=D$. This comes from the irreducibility assumption on $E$. If $K=E$, then $E \subset E^{\perp}$, which is equivalent to symmetry.

Theorem 402. Let $\pi$ be a Hermitian representation, and assume further that $\pi$ is nondegenerate. Let $\pi^{*}$ be the dual representation, and let $M=D^{*} / D$ be the quotient module.

Then an irreducible submodule $F$ in $M$ is symmetric if and only if $F+F^{\perp}$ is not dense in $M$, where

$$
F^{\perp}=\{\xi \in M: B(a, \xi, \eta)=0 \forall a \in \mathbb{A}, \eta \in F\} .
$$

Proof. It follows from the nondegeneracy that a subspace $S$ in $M$ is closed if and only if it is of the form $S=T^{\perp}$, or equivalently, $S=S^{\perp \perp}$. It follows from Lemma 3.2 that, whenever $F$ is a module in $M$, then $F^{\perp}$ is a module as well. This is because (Lemma 3.1) there is a one-to-one correspondence between the set of intermediate modules in $D^{*}$, and the set of modules in $M=D^{*} / D$ where the correspondence is defined by the quotient mapping $Q: D^{*} \rightarrow M$. Moreover,

$$
\left(Q^{-1}(F)\right)^{\perp}=Q^{-1}\left(F^{\perp}\right)
$$

holds for modules $F$ in $M$.

Working in the quotient $M$, we get

$$
\left(F+F^{\perp}\right)^{\perp}=F^{\perp} \cap F^{\perp \perp}=F^{\perp} \cap F .
$$


By the argument from the previous lemma, we must have $F^{\perp} \cap F=0$, or $F^{\perp} \cap F=F$. The density of $F+F^{\perp}$ is equivalent to $F^{\perp} \cap F=0$ by nondegeneracy. Finally, the possibility $F^{\perp} \cap F=F$ is equivalent to symmetry of the module $F$. The result follows.

Lemma 4.3. A Hermitian representation $\pi$ is nondegenerate if and only if $\pi=\pi^{* *}$. It follows that nondegenerate representations are closed.

Remark 4.4. Since we do not know whether or not, for general Hermitian representations $\pi$, the identity $\bar{\pi}=\pi^{* *}$ holds, there may be closed representations which are not nondegenerate.

Proof. We have the following inclusions for representations,

$$
\pi \subset \bar{\pi} \subset \pi^{* *} \text {. }
$$

It is known, in general [Po II], that $\pi^{* *}$ is contained in $\pi^{*}$ and it follows from this that $\pi^{* *}$ is Hermitian. If $\pi^{* *}=\bar{\pi}$, then it is nondegenerate.

The domains of the two representations $\pi^{*}$ and $\pi^{* *}$ are given as follows,

$$
\operatorname{dom}\left(\pi^{*}\right)=D^{*}=\bigcap\left\{\operatorname{dom}\left(\pi(a)^{*}\right): a \in \boldsymbol{A}\right\}
$$

and

$$
\operatorname{dom}\left(\pi^{* *}\right)=\cap\left\{\operatorname{dom}\left(\left(\left.\pi(a)^{*}\right|_{D^{*}}\right)^{*}\right): a \in A\right\} .
$$

A vector $u$ in $D^{*}$ is in $\left.\operatorname{dom}\left(\left.\left(\pi(a)^{*}\right)\right|_{D^{*}}\right)^{*}\right)$ iff there is some vector $w \in H$ such that

$$
\left(u, \pi(a)^{*} v\right)=(w, v) \quad \text { for all } \quad v \in D^{*} .
$$

But $D \subset D^{*}$, so, if $w$ exists, then $w=\pi^{*}(a) u$ and $B(a, u, v)=0$. If conversely $B(a, u, v)=0$ for all $v \in D^{*}$, then we may use the vector $\pi^{*}(a) u$ as $w$ in equation (4.5).

Corollary 4.5. Let $\pi$ be a nondegenerate Hermitian representation. Let $D$ be the domain of $\pi$ and $D^{*}$ that of $\pi^{*}$. Suppose the induced action on the quotient $D^{*} / D$ is irreducible.

Then $\pi$ is self-adjoint; in any case, it has no proper closed Hermitian extensions, i.e., it is maximal Hermitian.

Proof. It is assumed that the module $M=D^{*} / D$ is irreducible, and $M^{\perp}=0$ by the nondegeneracy assumption. So if $M \neq 0$, we cannot have $M \subset M^{\perp}$. Since $M$ does not contain any nontrivial submodules, there can be no proper extensions as specified in the corollary when $M \neq 0$. 


\section{§5. Selfadjoint Representations}

In this section we give criteria for selfadjointness of a representation. We consider decomposition theory for the induced modules, and apply this to representations of semisimple Lie algebras.

We are looking for essentially selfadjoint extensions $\rho$ of a given Hermitian representation $\pi$. Suppose a Hermitian extension $\rho$ is obtained from an intermediate subspace $E$ as in Lemma 3.1, i.e.,

$$
\rho(a):=\left.\pi^{*}(a)\right|_{E}, a \in \mathbb{A}
$$

and

$$
\operatorname{dom}(\rho)=E
$$

Then we say that $\rho$ is essentially selfadjoint if $\rho^{*}=\rho^{* *}$.

The argument from the proof of Lemma 3.2 gives the following additional information about extensions of a given Hermitian representation.

Proposition 5.1. Let $\pi$ be a Hermitian representation of an algebra $\mathbb{A}$ with involution, and let $\rho$ be a Hermitian extension of $\pi$.

(i) Let $E=\operatorname{dom}(\rho)$ and $E^{*}=\operatorname{dom}\left(\rho^{*}\right)$. Then

$$
E^{*}=E^{\perp}
$$

and $\rho$ is Hermitian iff $E \subset E^{\perp}$.

(ii) Let $E^{* *}=\operatorname{dom}\left(\rho^{* *}\right)$. Then

$$
E^{* *}=\left(E^{*}\right)^{\perp} \cap E^{*}
$$

It follows that a nondegenerate extension $\rho$ is essentially selfadjoint iff $E^{\perp}=E$.

Proof. We have $B(a, u, v)=\left(\pi^{*}(a) u, v\right)-\left(u, \pi(a)^{*} v\right)$ for all $a \in A, u, v \in D^{*}$.

If $u \in D^{*}$ and $v \in E$, then $B(a, u, v)=\left(\pi^{*}(a) u, v\right)-\left(u, \rho(a)^{*} v\right)$. Moreover, $u$ is in $\operatorname{dom}\left(\rho\left(a^{*}\right)^{*}\right)$ iff $\exists w \in H$ such that $\left(u, \rho\left(a^{*}\right) v\right)=(w, v)$ for $\forall v \in E$. Since $D \subset E$, we have $E^{*} \subset D^{*}$. So if further $u \in E^{*}$, then $w=\pi^{*}(a) u$, and $B(a, u, v)$ $=0$. The converse inclusion $E^{\perp} \subset E^{*}$ is immediate, and the proof of (5.3) is completed.

(ii) Formula (5.4) is from the proof of Lemma 3.2. If now $\rho$ is assumed nondegenerate, then $E^{* *}=E$. Combining (5.3) and (5.4), we then get $E=E^{* *}$ $=E^{\perp \perp} \cap E^{\perp}=E \cap E^{\perp}$. The equality $\rho^{* *}=\rho^{*}$ is then expressed as an equality between the respective domains, viz., $E=E^{\perp}$. 
In applications to representation theory for semisimple Lie algebras, the quotient $D^{*} / D$ is frequently finite-dimensional, see e.g., [JMo, Ch. 12]. Let $\mathrm{g}$ be a real semisimple Lie algebra, and let $G$ be a simply connected Lie group with $\mathrm{g}$ as its Lie algebra. Let $\pi_{0}$ be a representation of $\mathrm{g}$ with domain $D$ such that

$$
\left(\pi_{0}(x) u, v\right)=-\left(u, \pi_{0}(x) v\right), \forall x \in \mathfrak{g}, u, v \in D
$$

Let $\pi$ be the representation obtained from $\pi_{0}$ by extension to the universal enveloping algebra of $\mathrm{g}$. This enveloping algebra will be denoted $\boldsymbol{A}$ and it is the complex universal enveloping algebra of $\mathfrak{g}$ with involution, $a \rightarrow a^{*}$, determined by the conditions,

$$
x^{*}=-x, x \in \mathfrak{g}, \text { and } 1^{*}=1 .
$$

Then it follows from (5.5) and (5.6) that $\pi$ is Hermitian. Let $\pi^{*}$ denote the dual representation with domain $D^{*}$, and assume that the quotient space $D^{*} / D$ is finite-dimensional. Then it follows from semisimplicity of $\mathrm{g}$ that $\pi^{*}$ is semisimple on $D^{*} / D$, i.e., it breaks up as a direct sum

$$
M:=D^{*} / D=F_{1}+\cdots+F_{k}
$$

where each $F_{i}, i=1, \cdots, k$, is an irreducible module for the induced action of $\boldsymbol{A}$ on the quotient. When $F_{i}$ is viewed as a subspace of $M$, then $F_{i} \cap \sum F_{j}=0$ for $i \neq j$.

In studying Hermitian extensions $\rho$ of $\pi$ we may dispense with those modules $F_{i}$ from (5.7) where $F_{i} \cap F_{i}^{+} \neq 0$. They satisfy $F_{i} \subset F_{i}^{\perp}$ by Proposition 4.1 so they are Hermitian.

We shall restrict attention therefore to representations $\pi$ such that the components $F_{i}$ from (5.7) each satisfy $F_{i} \cap F_{i}^{\perp}=0$. It follows that then

$$
F_{i}+F_{i}^{+}=M
$$

so the induced representation from $\pi^{*}$ on $M$ is decomposable, and there is an explicit decomposition (5.8) for each of the irreducible components.

In checking whether or not a given intermediate submodule $E$ is symmetric, the following result is useful.

Theorem 5.2. Let $\pi$ be a nondegenerate Hermitian representation of an algebra $\boldsymbol{A}$ with involution. Let $D$ be the domain of $\pi$, and $D^{*}$ that of $\pi^{*}$. Let $S$ be a subset of $\mathbb{A}$ such that $B(a, v, u)=0$ for all $a \in S, u, v \in D^{*}$. Suppose $\mathbb{A}$ is the smallest subalgebra containing $S$ (i.e., $\boldsymbol{A}$ is generated as an algebra by $S$ ). 
Then it follows that $\pi$ is selfadjoint.

Proof. For $a, b \in \mathbb{A}$ and $u, v \in D^{*}$, we have

$$
B(a b, u, v)=B\left(a, \pi^{*}(b) u, v\right)+B(b, u, \pi(a) * v) .
$$

The right hand side vanishes identically if it is further assumed that $a, b \in S$. Since $S$ generates $A$ as an algebra, we get $B(a, u, v)=0$ for all $a \in A, u, v \in D^{*}$. So $D^{*}$ is symmetric. We have $D^{*} \subset D^{* \perp}$. But $D^{* \perp}=D^{* *}$ by Proposition 5.1. Since $\pi$ is assumed nondegenerate, $D^{* *}=D$, so $D^{*}=D$ which is equivalent to selfadjointness of $\pi$.

Remark 5.3. The assumptions of the theorem are satisfied in a class of representations arising from semisimple real $\mathbb{L i e}$ algebras. Let $\mathrm{g}$ be a semisimple Lie algebra with Cartan decomposition, $\mathfrak{g}=\mathfrak{i}+\mathfrak{p}$, with the Killing form negative definite on and positive definite on $\mathfrak{p}$, and

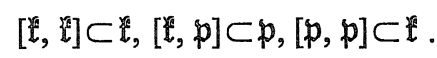

Let $\mathbb{A}$ be the complex universal enveloping algebra of $\mathrm{g}$.

Assume

(i) $[\mathfrak{p}, \mathfrak{p}]=\mathbb{1}$

and

(ii) $B(x, u, v)=0$ for $\forall x \in \mathfrak{p}, u, v \in D^{*}$.

Then the theorem applies and we conclude that $\pi$ is essentially selfadjoint, i.e., $\bar{\pi}=\pi *$.

The argument from the proof of the theorem shows that $D^{*}$ is symmetric, i.e., $D^{*} \subset D^{* \perp}$. It follows that $D^{* *}=D^{*}$. But if $\pi$ is obtained, as above, by extension from a Lie algebra representation $\pi_{0}$ satisfying (5.5), then it follows that $\bar{\pi}=\pi^{* *}$.

Using known results for finite-dimensional Hilbert spaces, and subspaces in generic position [Su], the previous discussion yields information about $D^{*} / D$ for a nontrivial class of representation:

Scholium A. Let $\pi$ be a nondegenerate Hermitian representation such that the module $M=D^{*} / D$ is finite-dimensional with decomposition (5.7) for $k=2$. Suppose further that any two of the four subspaces $F_{1}, F_{1}^{\perp}, F_{2}, F_{2}^{\perp}$ have trivial intersection. Then it follows that $F_{1}$ is the graph of a linear isomorphism of $F_{2}$ onto $F_{2}^{\perp}$.

Remark. An analogous more general result is available for nondegenerate 
Hermitian representations such that the module $M=D^{*} / D$ is finite-dimensional with decomposition (5.7) with arbitrary $k \geq 2$. The proof of this is again based on ideas from [Su], but we shall postpone details to a later publication, as they are not needed for the present paper.

When this is applied to $s l_{2}(\mathbb{R})$, Theorem 5.2 allows us to decide the symmetry question for Hermitian representations and intermediate modules from the consideration of a single quadratic form.

Let $\mathrm{g}=s l_{2}(\mathbb{R})$. Let $D$ be a vector space with inner product, and let $H$ be the corresponding Hilbert space completion. A Hermitian representation $\pi$ of $\mathrm{g}$ is given uniquely by a pair of operators $h_{ \pm}: D \rightarrow D$ such that:

(i) $\left(h_{+} u, v\right)=\left(u, h_{-} v\right), \quad u, v \in D$.

(ii) The operator $h_{0}:=-\frac{1}{2}\left[h_{+}, h_{-}\right]$satisfies $\left[h_{0}, h_{ \pm}\right]= \pm h_{ \pm}$.

For such a pair, consider the corresponding representation $\pi$, and the quotient $D^{*} / D$ where $D=\operatorname{dom}(\pi)$ and $D^{*}=\operatorname{dom}\left(\pi^{*}\right)$. On $D^{*}$, we define the single quadratic form

$$
Q(u, v):=\left(h_{+}^{*} u, v\right)-\left(u, h_{-}^{*} v\right), \quad u, v \in D^{*}
$$

Finally, let $E$ be an intermediate submodule, i.e., $D \subset E \subset D^{*}$.

We have

Corollary 5.4. Let $E$ and $Q$ be as above. Then $E$ is symmetric iff

(iii) $Q(u, v)=0$ for all $u, v \in E$.

Proof. This result is immediate from Theorem 5.2. The important step is that condition (iii) above implies

$$
B(a, u, v)=0 \forall a \in \mathfrak{U}_{c}(\mathrm{~g}), \quad u, v \in E
$$

where $B(a, u, v)$ is given by (2.9).

Theorem 5.2 has the further advantage of facilitating the computation of the group $G(\pi)$ of "unitary" transformations where "unitarity" is defined with respect to the (nonpositive definite) quadratic forms $\{B(a, \cdot): a \in \mathbb{A}\}$.

Let $\mathbb{A}$ be given, and let $\pi$ be a nondegenerate Hermitian representation of $A$ with domain $D$. Let $D^{*}$ denote the domain of $\pi^{*}$, and let $M$ denote the induced (quotient) module $M=D^{*} / D$. We shall denote by $G(\pi)$ the group of linear transformations, $T: M \rightarrow M$ such that

(i) $T$ is invertible, 
(ii) $B(a, T \xi, T \eta)=B(a, \xi, \eta), \forall a \in \mathbb{A}, \xi, \eta \in M$

and

(iii) $\pi^{*}(a) T \xi=T \pi^{*}(a) \xi, \forall a \in A, \quad \xi \in M$;

where we have used the notation $B(a, \cdot)$, and $\pi^{*}(a)$, for the quadratic forms, resp. the operators, which are induced from the corresponding forms, resp. operators, on $D^{*}$ by passage to the quotient $D^{*} / D=M$.

Note also that, in fact, (i) follows from (ii)-(iii) by virtue of the nondegeneracy assumption.

Corollary 5.5. Let $\pi$ be as in Theorem 5.2, and let $S$ be a set of generators for the given algebra $\mathbb{A}$. Then a linear transformation $T: M \rightarrow M$ satisfies (ii)(iii) for all $a \in A$ iff the two conditions are assumed to hold only for $a$ in $S$.

Proof. Based on (3.4) above: It is immediate that (iii) follows for all $a \in \mathbb{A}$ when it is assumed initially only for $a \in S$. Also note that the quadratic forms $\left\{B\left(a,{ }^{\circ}\right): a \in S\right\}$ form a nondegenerate family on $M$. So if $\xi \in M$ and $B(a, \xi, \eta)=0$ for all $a \in S$ and all $\eta \in M$, it follows that $\xi=0$. A standard fact on quadratic forms implies invertibility of $T$ under consideration.

To extend the validity of (ii) from $S$ to all of $A$, we proceed as follows:

We need only consider the multiplicative property of $a \rightarrow B\left(a,{ }^{\circ}\right)$, since linearity is given. The problem reduces to showing that (ii) must hold for the product $c=a b$ whenever it is assumed for the two elements $a$ and $b$ separately. We now make the assumption (ii) for the individual elements $a, b$, and consider $\zeta, \eta \in M$. We have, using (3.4) twice:

$$
\begin{aligned}
B(c, T \xi, T \eta) & =B\left(a, \pi^{*}(b) T \xi, T \eta\right)+B\left(b, T \xi, \pi(a)^{*} T \eta\right) \\
& =B\left(a, T \pi^{*}(b) \xi, T \eta\right)+B\left(b, T \xi, T \pi(a)^{*} \eta\right) \\
& =B\left(a, \pi^{*}(b) \xi, \eta\right)+B\left(b, \xi, \pi(a)^{*} \eta\right) \\
& =B(c, \xi, \eta)
\end{aligned}
$$

where we used (iii) in the second step of the computation. This is the desired formula.

Remarks 5.6. We consider transformations $T: M \rightarrow M$ since such transformations $T$ do not always lift to transformations $\widetilde{T}: D^{*} \rightarrow D^{*}$ satisfying $\widetilde{T}(D) \subset D$, and the two invariance conditions on $D^{*}$. However, it is true that the condition $\tilde{T}(D) \subset D$ is implied by the invariance conditions (i)-(iii) placed on transformations $\widetilde{T}: D^{*} \rightarrow D^{*}$, i.e.,

(i) $\widetilde{T}$ is invertible on $D^{*}$; 
(ii) $\quad B(a, \tilde{T} u, \tilde{T} v)=B(a, u, v)$;

(iii) $\pi^{*}(a) \widetilde{T} u=\widetilde{T} \pi^{*}(a)$ modulo $D$, assumed for $a \in A, \quad u, v \in D^{*}$.

We give the proof: By nondegeneracy, we have

$$
D=\left\{u \in D^{*}: B(a, u, v)=0, \forall a \in \mathbb{A}, v \in D^{*}\right\} .
$$

If $\widetilde{T}$ satisfies (i) -(iii) ${ }^{\sim}$, and $u \in D$ is given, then $B(a, \widetilde{T} u, v)=B\left(a, u, \widetilde{T}^{-1} v\right)=0$ for all $a \in \mathbb{A}$ and all $v \in D^{*}$. It follows that $\widetilde{T} u \in D$ as claimed. So $\tilde{T}$ does pass to the quotient $D^{*} / D=M$. But there are examples which show that the lifting problem does not always have solutions.

Examples 5.7. The groups $G=G(\pi)$ constructed from nondegenerate Hermitian representations include as examples the classical reductive Lie groups $U(p, q)$, cf. [He], but some infinite-dimensional analogues of the $U(p, q)$ groups are also included in the class of groups $\{G(\pi)\}$.

The following easy example shows how $U(1,1)$ arises. Let $H=L^{2}(0,1)$, and $D=C_{c}^{\infty}(0,1)=$ all smooth functions on the unit-interval which vanish of infinite order at both endpoints. Let $\mathbb{A}=\mathbb{C}[x]$, and let $\pi(x)=d / d t$. Then $\pi$ defines (by extension to $\mathbb{A}$ ) a nondegenerate Hermitian representation of the singly generated algebra $A$, and the group $G(\pi)$ is given in terms of $U(1,1)$ where

$$
U(1,1)=\left\{\left[\begin{array}{cc}
\alpha & \beta \\
\bar{\beta} & \bar{\alpha}
\end{array}\right]:|\alpha|^{2}-|\beta|^{2}=1\right\}
$$

is the familiar hyperbolic (conformal) group of complex 2 by 2 matrices, i.e., the invariance group for the quadratic form

$$
B=z_{1} \bar{w}_{1}-z_{2} \bar{w}_{2} .
$$

By calculus, this form agrees with the quadratic form $B(x, \cdot)$ on $D^{*} / D=M$, and $M \supset \mathbb{C}^{2}$. We sketch the details below:

Inclusion of higher order terms is needed in a complete coordinatization of the space $D^{*} / D$. In fact, an iteration of formula (3.4) shows that $D^{*} / D$ may be identified with the direct limit space $\bigsqcup_{0}^{\infty} \mathbb{C}^{2}$ consisting of vectors $z=\left(z^{(n)}\right)_{n=0}^{\infty}$ with $z^{(n)} \in \mathbb{C}^{2}$ and nonzero for at most a finite number of values of $n$ (depending on $z$ ). Let $B$ be the symplectic form on $\mathbb{C}^{2}$ which is given in (5.11). We have a complete family of quadratic forms $\left\{B_{m}: m=1,2, \cdots\right\}$ on $D^{*} / D$ given by the formula

$$
B_{m}(z, z)=\sum_{k=0}^{m} B\left(z^{(k)}, z^{(m-k)}\right)
$$


for $m=1,2, \cdots$. So a more precise description of $G(\pi)$ involves a product construction from an infinite number of copies of the group $U(1,1)$.

The above remarks apply more generally to Hermitian representations of singly generated algebras with involution.

Rao [Ra] showed that the two-parameter unitary dual of the simply connected covering group of $S L_{2}(\mathbb{R})$ may be constructed from integrable (selfadjoint) extensions of a single nondegenerate Hermitian representation $\pi$ of $\mathfrak{U}_{C}\left(\mathfrak{d}_{2}(\mathbb{R})\right)$. It follows from our Theorem 6.1 (in the next section) that $D^{*}$, for these examples, may be computed from singly generated algebras, and the groups $G(\pi)$, for the singly generated algebras in the Rao-examples, turn out to be subgroups of the Lie group $U(2,2)$, or, more generally, a direct product construction involving an infinite number of copies of the group $U(2,2)$.

\section{§6. Relatively Bounded Representations}

In this section, we consider a class of representations where the induced modules may be studied in terms of a restriction to a certain subalgebra of the given Hermitian representation.

Consider a given Hermitian representation $\pi$ of an algebra $\mathbb{A}$ and assume that the domain $D=\operatorname{dom}(\pi)$ is dense in the underlying Hilbert space $H$. Let $\pi^{*}$ be the adjoint representation. We shall consider further the induced quotient module $D^{*} / D$ where

$$
D^{*}=\operatorname{dom}\left(\pi^{*}\right)=\bigcap\left\{\operatorname{dom}\left(\pi(a)^{*}\right): a \in \mathbb{A}\right\} .
$$

For the purpose of understanding $D^{*} / D$ (and computing the answer!), it is practical to reduce the family of operators $\{\pi(a): a \in \mathbb{A}\}$ by passing to a subalgebra $\mathbb{A}_{0} \subset \mathbb{A}$. In the event (considered here) where $\mathbb{A}$ is the universal enveloping algebra of a given semisimple real Lie algebra $\mathfrak{g}$ with Cartan decomposition, $\mathfrak{g}=\mathfrak{i}+\mathfrak{p}$ with $\mathfrak{t}$ and $\mathfrak{p}$ satisfying

$$
[\mathfrak{l}, \mathfrak{l}] \subseteq \mathfrak{l},[\mathfrak{i}, \mathfrak{p}] \subseteq \mathfrak{p},[\mathfrak{p}, \mathfrak{p}] \subseteq \mathfrak{t},
$$

we shall consider this possibility

$$
\mathbb{A}=\mathfrak{U}_{C}(\mathfrak{g}) \text { and } \mathbb{A}_{0}=\mathfrak{U}_{C}(\mathfrak{t}) .
$$

Let $K$ be the (nondegenerate) Killing form of $\mathrm{g}$ and pick a basis $\left\{x_{i}\right\}$ for $\mathrm{g}$ such that $\left\{x_{i}: 1 \leq i \leq r\right\}$ is a basis for $\mathfrak{t}$, and $\left\{x_{j}: r<j \leq r+s\right\}$ is a basis for $\mathfrak{p}$ satisfying 


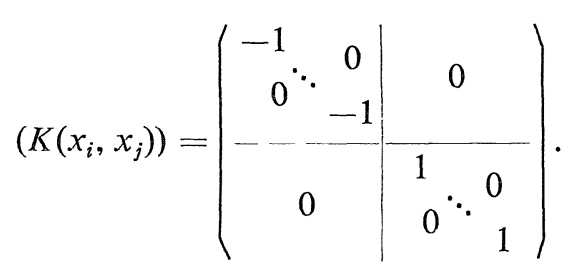

The corresponding Casimir element $\Omega$ in $A$ is given by

$$
\Omega=-\sum_{1}^{r} x_{i}^{2}+\sum_{r+1}^{s} x_{j}^{2}
$$

A given Hermitian representation $\pi$ is said to be quasi-simple if $\pi(\Omega)$ is a scalar. We shall prove the following

Theorem 6.1. Let $\mathrm{g}$ be a semisimple real Lie algebra, and let $\pi$ be a Hermitian representation of $\boldsymbol{A}=\mathfrak{u}_{\boldsymbol{c}}(\mathfrak{g})$. Let $\mathfrak{g}=\mathfrak{l}+\mathfrak{p}$ be a Cartan decomposition and $\boldsymbol{A}_{0}=\mathfrak{U}_{\boldsymbol{C}}(\mathfrak{\mathfrak { t }})$. If $\pi$ is quasi-simple, then it follows that

$$
D^{*}(\boldsymbol{A})=D^{*}\left(\mathbb{A}_{0}\right) .
$$

The following observation is needed in the proof:

Lemma 6.2. Let $\pi, \mathfrak{g}$ be as above, and choose a basis $\left\{x_{i}\right\}_{i=1}^{r+s}$ as in (6.3). Then

$$
\sum_{r+1}^{r+s}\left\|\pi\left(x_{j}\right) u\right\|^{2}=\sum_{1}^{r}\left\|\pi\left(x_{i}\right) u\right\|^{2}+\lambda\|u\|^{2}, u \in D
$$

where $\lambda=-\pi(\Omega)$.

Proof. For all $u \in D$, we have

$$
\begin{aligned}
-\lambda\|u\|^{2} & =(u, \pi(\Omega) u) \\
& =-\sum_{1}^{r}\left(u, \pi\left(x_{i}\right)^{2} u\right)+\sum_{r+1}^{r+s}\left(u, \pi\left(x_{j}\right)^{2} u\right) \\
& =\sum_{1}^{r}\left\|\pi\left(x_{i}\right) u\right\|^{2}-\sum_{r+1}^{r+s}\left\|\pi\left(x_{j}\right) u\right\|^{2}
\end{aligned}
$$

which is the desired formula.

Remark 6.3. We say that the Lie algebra $g$ is relatively bounded by the subalgebra $\mathfrak{t}$ since (6.5) implies the following operator theoretic relative bound: For every $y \in \mathrm{g}$, there is a constant $C$ such that

$$
\|\pi(y) u\| \leq C\left[\sum_{1}^{r}\left\|\pi\left(x_{i}\right) u\right\|+\|u\|\right]
$$


We also note that the estimate (6.6) may be strengthened to give analytic domination. We refer to [Ne], [JMo, Ch. 12], and [GJ, Thm. 2.1] for details on analytic domination: We get that every operator $\pi(y)$ for $y \in \mathfrak{g}$ is analytically dominated by $\{\pi(x): x \in \mathbb{f}\}$, and, moreover, the family $\left\{\pi\left(x_{i}\right): 1 \leq i \leq r+s\right\}$ is analytically dominated by the smaller family $\left\{\pi\left(x_{i}\right): 1 \leq i \leq r\right\}$.

Defimmition $6_{0} 4$. A family of linear mappings $\left\{\beta_{i}: i=0,1, \cdots, r\right\}$ from $\mathfrak{p}$ into the algebra $\mathfrak{B}(H)$ of all bounded linear operators on $H$ is said to be a derived representation if the following two conditions hold:

$$
\pi(y)=\beta_{0}(y)+\sum_{1}^{r} \beta_{i}(y) \pi\left(x_{i}\right)
$$

and

$$
\left[\pi(x), \beta_{i}(y)\right]=\beta_{i}([x, y])
$$

valid for $y \in \mathfrak{p}, x \in$ and $0 \leq i \leq r$ where $\mathfrak{g}=\mathfrak{l}+\mathfrak{p}$ is a given Cartan decomposition and the basis $\left(x_{i}\right)$ is specified as in (6.3).

We note that (6.7) is a pointwise operator identity on the given dense domain $D \subset H$, while (6.8) must be interpreted in the sense of quadratic forms:

$$
\left(\pi(x)^{*} u, \beta_{i}(y) v\right)-\left(u, \beta_{i}(y) \pi(x) v\right)=\left(u, \beta_{i}([x, y]) v\right)
$$

for all $x \in \mathfrak{l}, y \in \mathfrak{p}$, and all pairs of vectors $u \in \operatorname{dom}\left(\pi(x)^{*}\right), v \in D$.

Corollary 6.5. Every quasi-simple Hermitian representation $\pi$ of a semisimple real Lie algebra has a bounded derived representation.

Proof. Apply Lemma 6.2. For further details, see also the proof in [Jo (a), Thm. 9.2]. (The Hermitian property of $\pi$ is used in a crucial way in the proof.)

Corollary 6.6. Let $\pi, \mathrm{g}$ be a representation as specified above, and let $\left\{\beta_{i}\right\}_{i=0}^{r}$ be a derived representation defined relative to some basis $\left\{x_{i}\right\}$ for as specified in (6.3). Then we have the following inclusion of operator domains:

$$
\bigcap_{i=1}^{r} \operatorname{dom}\left(\pi\left(x_{i}\right)^{*}\right) \subset \operatorname{dom}\left(\pi(y)^{*}\right)
$$

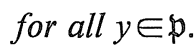

Proof. Let $u \in \bigcap_{i} \operatorname{dom}\left(\pi\left(x_{i}\right)^{*}\right)$ and $v \in D=\operatorname{dom} \pi$. Then

$$
\begin{aligned}
(u, \pi(y) v) & =\sum_{i}\left(u, \beta_{i}(y) \pi\left(x_{i}\right) v\right) \\
& =\sum_{i}\left(\pi\left(x_{i}\right)^{*} u, \beta_{i}(y) v\right)-\sum_{i}\left(u, \beta_{i}\left(\left[x_{i}, y\right]\right) v\right) .
\end{aligned}
$$


Using boundedness of the derived representation, we conclude that $u \in$ dom $\left(\pi(y)^{*}\right)$, and that

$$
x(y)^{*} u=\sum_{i} \beta_{i}(y)^{*} \pi\left(x_{i}\right)^{*} u+\sum_{i} \beta\left(\left[y, x_{i}\right]\right)^{*} u .
$$

It follows from the construction of the derived representation $\left(\beta_{i}\right)$ in Corollary 6.5 (from Lemma 6.2) that each bounded operator $\beta_{i}(y), y \in \mathfrak{p}, i=0,1, \cdots, r$ may be chosen to map the domain $D$ into the intersection $\cap\{\operatorname{dom}(\overline{\pi(x)}): x \in \mathrm{g}\}$. It follows further from this, and iteration of (6.6), that each $\beta_{i}(y)$ maps $D$ into the domain of the closure representation $\bar{\pi}$, i.e.,

$$
\operatorname{dom}(\bar{\pi})=\bigcap\{\operatorname{dom}(\overline{\pi(a)}): a \in A\} .
$$

The operators $\beta_{i}(y)$ are smooth operators.

We now turn to the proof of Theorem 6.1 which is based on an iteration of the two formulas (6.7) and (6.8) in Definition 6.4. For the purpose of the present proof we shall adopt the following simplifying notation for operator monomials and commutators. We shall work with multi-indices $\alpha=\left(\alpha_{1}, \alpha_{2}, \cdots\right.$, $\left.\alpha_{m}\right)$ with $\alpha_{i}=1,2, \cdots$. For a given family of elements $\left(y_{i}\right)$ in $\mathfrak{p}$, we introduce the monomials

$$
y(\alpha):=y_{\alpha_{1}} y_{\alpha_{2}} \cdots y_{\alpha_{m}} .
$$

But it shall be convenient to denote such a monomial by $y^{m}$ when the specific form of the expression is not needed, other than the degree $m$. We shall also adopt the similar notation for monomials formed from elements $\left(x_{i}\right)$ in a given basis for $\mathfrak{f}$. We also recall that the enveloping algebra has a basis consisting of monomials by the Birkhoff-Witt theorem [He, Ch. II, §2, p. 102].

It follows from Corollary 6.5 and induction that higher degree analogues of the bounded derived representation may be found. In multi-index notation (with noncommutative monomials as specified above), it takes the following form:

$$
\pi\left(y^{m}\right)=\sum \beta_{n}\left(y^{m}\right) \pi\left(x^{n}\right)
$$

where $y^{m}$ denotes a degree $m$ monomial formed from elements in $\mathfrak{p}$, and $x^{n}$ denotes a degree $n$ monomial formed from the given basis elements $x_{1}, \cdots, x_{r}$ in $\mathfrak{f}$. It is also understood that the summation in (6.10) is extended over all possible multi-indices of degree $n$ where $n \leq m$.

We now turn to the iteration of the second formula (6.8) from Definition 6.4. It is clear that the desired conclusion (6.4) from Theorem 6.1 will then follow. 
We need to establish, by induction, the following commutator formula valid for monomials $y^{m}$ over $\mathfrak{p}$, and monomials $x^{n}$ over $\mathfrak{i}$ :

$$
\left[\pi\left(x^{n}\right), \beta\left(y^{m}\right)\right]=\sum \pi\left(x^{n-1}\right) \beta\left(y^{m}\right)
$$

where the summation is extended again over all possible monomials which occur upon carrying through the combinatorics of the iterated commutators, and keeping in mind the reduction $[x, y] \in \mathfrak{p}$ for all $x \in \mathbb{f}$ and all $y \in \mathfrak{p}$, cf. (6.1) above. We leave the further details of the combinatorics to the reader. (It is very analogous to the combinatorial proof of the Birkhoff-Witt theorem.) We restrict attention instead to consideration of operator-domains. We prove, as in Corollary 6.5 above, the following inclusion of domains,

$$
\bigcap_{n \leq m} \operatorname{dom}\left(\pi\left(x^{n}\right)^{*}\right) \subset \operatorname{dom}\left(\pi\left(y^{m}\right)^{*}\right) .
$$

Consider a pair of vectors $u \in \bigcap_{n \leq m} \operatorname{dom}\left(\pi\left(x^{n}\right)^{*}\right)$ and $v \in D$. As in the proof of Corollary 6.6, we now have

$$
\begin{aligned}
\left(u, \pi\left(y^{m}\right) v\right) & =\sum\left(u, \beta_{n}\left(y^{m}\right) \pi\left(x^{n}\right) v\right) \\
& =\sum\left(u, \pi\left(x^{n}\right) \beta_{n}\left(y^{m}\right) v\right)-\sum\left(u,\left[\pi\left(x^{n}\right), \beta_{n}\left(y^{m}\right)\right] v\right) .
\end{aligned}
$$

But, for the commutator in the last summation term, we have just established the expression,

$$
\left[\pi\left(x^{n}\right), \beta_{n}\left(y^{m}\right)\right]=\sum \pi\left(x^{n-1}\right) \beta\left(y^{m}\right),
$$

and the terms in this summation are included in the general form

$$
\sum \pi\left(x^{n}\right) \beta\left(y^{s m}\right)
$$

with a suitable redefinition of the nonzero terms $\beta\left(y^{m}\right)$ of bounded operator monomials. When all the terms are collected in a single summation, we get

$$
\begin{aligned}
\left(u, \pi\left(y^{m}\right) v\right) & =\sum\left(u, \pi\left(x^{n}\right) \beta\left(y^{m}\right) v\right) \\
& =\sum\left(\pi\left(x^{n}\right) * u, \beta\left(y^{m}\right) v\right)
\end{aligned}
$$

since $u \in \operatorname{dom}\left(\pi\left(x^{n}\right)^{*}\right)$. It follows then, from boundedness of $\beta\left(y^{m}\right)$, that $u \in \operatorname{dom}\left(\pi\left(y^{m}\right)^{*}\right)$, and that

$$
\pi\left(y^{m}\right) * u=\sum \beta\left(y^{m}\right) * \pi\left(x^{n}\right) * u
$$

generalizing formula (6.9) above from the proof of Corollary 6.6.

It follows that

$$
D^{*}\left(A_{0}\right) \subset D^{*}(A)
$$


where

$$
\begin{aligned}
& D^{*}\left(\boldsymbol{A}_{0}\right)=\bigcap\left\{\operatorname{dom}\left(\pi\left(a^{*}\right)\right): a \in \boldsymbol{A}_{0}\right\}, \\
& D^{*}(\boldsymbol{A})=\cap\left\{\operatorname{dom}\left(\pi(a)^{*}\right): a \in \boldsymbol{A}\right\},
\end{aligned}
$$

and $\boldsymbol{A}$, resp. $\boldsymbol{A}_{0}$, are given in (6.2) above. Since the other inclusion from (6.4) is trivial the proof of Theorem 6.1 is concluded.

We have two corollaries.

Corollary 6.7. Let the Lie algebra $\mathrm{g}$ be semisimple with Cartan decomposition $\mathfrak{g}=\mathfrak{t}+\mathfrak{p}$, and let $\pi$ be a Hermitian quasi-simple representation of $\mathfrak{u}_{c}(\mathfrak{g})$. Then $\pi$ is essentially selfadjoint if and only if the restriction to $\mathfrak{U}_{C}(\mathfrak{l})$ is essentially selfadjoint.

Corollary 6.8. Let $\pi$ and $\mathfrak{g}$ be as above. If $\operatorname{dim} \mathfrak{i}=1$ (e.g., $\mathfrak{g} \simeq s h_{2}(\boldsymbol{R})$ ), then it follows that $\pi$ is essentially selfadjoint if and only if it is integrable.

Proof. We say that $\pi$ is integrable if there is a strongly continuous unitary representation $R$ of the simply connected Lie group $G$ with Lie algebra isomorphic to $\mathrm{g}$ such that $d R=\bar{\pi}$ where $d R$ denotes the differential of $R$. It is well known that $d R$ is selfadjoint, see [PoI] and [Jo (c)]. So if $\pi$ is assumed integrable, it must be essentially selfadjoint, cf. also [Po II, Sect. 4] an [Ra, Corollary A1].

Assume now that $\pi$ is given and assumed essentially selfadjoint as a representation of $\mathfrak{u}_{c}(\mathfrak{g})$. Pick a basis vector $x_{0}$ for the one-dimensional Cartan component $\mathfrak{l}$. Since $\pi$ is quasi-simple, it follows from Nelson's theorem [Ne] that $\pi$ is integrable if and only if the single operator $\pi\left(x_{0}\right)$ is essentially selfadjoint. But the algebra $\boldsymbol{A}_{0}=\mathfrak{u}_{\boldsymbol{c}}(\mathfrak{t})$ is singly generated and the restriction of $\pi$ to $\boldsymbol{A}_{0}$ is essentially selfadjoint by Theorem 5.2. It then follows from [Po I, Lemma 3.2] that $\pi\left(x_{0}\right)$ is essentially selfadjoint so Nelson's theorem applies.

Remarks and Examples 6.9. We conjecture that the conclusion in Corollary 6.8 holds in general even when the restriction $\operatorname{dim} \mathfrak{f}=1$ is removed. It would follow then that every quasi-simple selfadjoint representation of a semisimple Lie algebra $\mathfrak{g}$ (or more precisely of $\mathfrak{U}_{c}(\mathfrak{g})$ ) is integrable. The conjecture is consistent with the recent examples (due to Kostant and $\emptyset$ rsted [KØ]) of families of nonintegrable representations of $s a(p, q)$ for $p, q \geq 3$ and $p+q$ odd. These representations are not selfadjoint since they do not have nonzero $K$-finite vectors, cf. Theorem 6.1 above.

In Theorem 6.1 and Corollaries 6.7-6.8 above, we assumed that the given Hermitian representation $\pi$ is also quasi-simple, i.e., that $\pi(\Omega)$ is a scalar 
times the identity operator where $\Omega$ is the Casimir element given in formula $\left(6.3^{\prime}\right)$. We showed in [Jo (a)], in the case of $\mathrm{g}=1 \ell_{2}(\boldsymbol{R})$ how this assumption can be slightly relaxed, but it cannot be removed.

Let $\boldsymbol{A}=\mathfrak{U}_{\boldsymbol{c}}\left(s \ell_{2}(\mathbb{R})\right)$. Then Schmüdgen [Sc] constructed a specific selfadjoint representation $\pi$ of $\mathbb{A}$ which is not integrable. In his example, the operator $\pi(\Omega)$ is not a scalar times the identity operator; in fact, it is unbounded, and affiliated with the commutant von Neumann algebra $\mathscr{M}_{\pi}=\pi(\mathcal{A})^{\prime}$ studied by Powers [Po I]. Combining two results of Powers [Po I, Lemma 3.2 and 4.6] with the integrability theorem of Nelson [Ne], we conclude that, for Schmüdgen's representation $\pi$, the conclusion (6.4) in Theorem 6.1 fails. It is immediate also that the conclusions in Corollaries 6.7 and 6.8 fail for Schmüdgen's representation $\pi$.

While the problem from Remark 4.4 remains open for general Hermitian representations, the identity $\pi=\pi^{* *}$ can be shown to hold for the special closed Hermitian representations $\pi$ considered in Section 6 above. Since this result is not needed in the present paper, the proof will be postponed to a future article. Even though the identity $\pi=\pi^{* *}$ may possibly fail for some (pathological) closed Hermitian representations, it would be interesting to focus on special classes of representations (such as those in Sect. 6) where it does hold.

\section{References}

[Ar] Araki, H., Indecomposable representations with invariant inner product-a theory of the Gupta-Bleuler triplet, Commun. Math. Phys. 97 (9185), 149-159.

[Arv] Arveson, W.B., An addition formula for the index of semigroups of endomorphisms of $B(H)$, Pacific J. Math., 137 (1989), 19-36.

[CP] Chari, V. and Pressley, A., Unitary representations of the Virasoro algebra and a conjecture of Kac, Compositio Math., 67 (1988), 315-342.

[DS] Dunford, N. and Schwartz, J.T., Linear Operators, Part II, Interscience Publisher (J. Wiley), New York, 1988.

[GJ] Goodman, F.M. and Jorgensen, P.E.T., Lie algebras of unbounded derivations, $J$. Funct. Anal., 52 (1983), 369-384.

[GW] Goodman, Roe and Wallach, N.R., Projective unitary positive-energy representations Diff $\left(S^{1}\right)$, J. Funct. Anal., 63 (1985), 299-321.

[Ha] Hannabuss, K.C., Selfadjoint operators in indefinite inner product spaces, Quart. J. Math. (2), 39 (1988), 333-348.

[He] Helgason, S., Differential Geometry, Lie Groups and Symmetric Spaces, Academic Press, New York, 1978.

[Helt] Helton, J.W., Unitary operators on a space with an indefinite inner product, $J$. Funct. Anal., 6 (1970), 412-440.

[In] Inoue, A., Selfadjointness of *-representations generated by positive linear functionals, Proc. Amer. Math. Soc., 93 (1985), 643-647.

[Jo(a)] Jorgensen, P.E.T., Representations of differential operators on a Lie group, and 
conditions for a Lie algebra of operators to generate a representation of the group, J. d' Analyse Math., 43 (1983/84), 251-288.

[Jo(b)] Jorgensen, P.E.T., Analytic continuation of local representations of a Lie group, Pacific J. Math. (2), 124 (1986), 397-408.

[Jo(c)] - Operators and Representation Theory, North Holland Publ. Co. Math. Stud. 147, Amsterdam, 1988.

[JMo] Jorgensen, P.E.T. and Moore, R.T., Operator Commutation Relations, D. Reidel Publ. Co. (Kluwer), Dordrecht-Boston, 1984.

[JMu] Jorgensen, P.E.T. and Muhly, P.S., Selfadjoint extensions satisfying the Weyl operator commutation relations, J. $d^{\prime}$ Analyse Math., 37 (1980), 46-99.

[KO] Kostant, B. and $\emptyset$ rsted, B., Minimal nilpotent co-adjoint orbits, Reprint 1987 (MIT/Odense).

[Kr] Krein, M.G., Introduction to the geometry of indefinite J-spaces and to the theory of operators in those spaces, Amer. Math. Soc. Transl., 93 (1970), 103-176.

[La] Lassner, G., Topological algebras of operators, Rep. Math. Phys., 3 (1972), 279-293.

[Na] Nakagami, Y., Spectral analysis in Krein-spaces, Publ. RIMS, Kyoto Univ., 24 (†3) (1988).

[Ne] Nelson, E., Analytic vectors, Ann. Math., 70 (1959), 572-615.

[vN] von Neumann, J., Zur Algebra der Funktionaloperationen und Theorie der Normalen Operatoren, Math. Ann., 102 (1929/30), 370-427.

[Ot] Ota, S., Certain operator algebras induced by *-derivations on $\mathrm{C}^{*}$-algebras on an indefinite inner product space, J. Funct. Anal., 30 (1978), 238-244.

[Ph] Phillips, R.S., The extension of dual subspaces invariant under an algebra, in Proc. Internat. Symp. Lin. Spaces, Jerusalem, Academic Press, New York, 1961 (pp. 366398).

[Po] Powers, R.T., Selfadjoint algebras of unbounded operators, I: Commun. Math. Phys. 21 (1971), 85-124; II: Trans. Amer. Math. Soc., 187 (1974), 261-293.

[PP] Powers, R.T. and Price, G., Continuous spatial semigroups of $*$-endomorphisms of B(H), Preprint, USNA 1988, to appear in Amer. Math. Soc. Transacticons.

[Ra] Rao, R.R., Unitary representations defined by boundary conditions $\left(\mathcal{S} \ell_{2}(R)\right)$, Acta Math., 139 (1977), 185-216.

[Sc] Schmüdgen, K., Personal communication (1986).

[Su] Sunder, V.S., $\quad N$ subspaces, Canad J. Math., 40 (1988), 38-54.

[Ta] Takesue, K., Standard representations induced by positive linear functionals, Mem. Fac. Sci. Kyushu Univ., Ser. A, 37 (1983), 211-225. 
